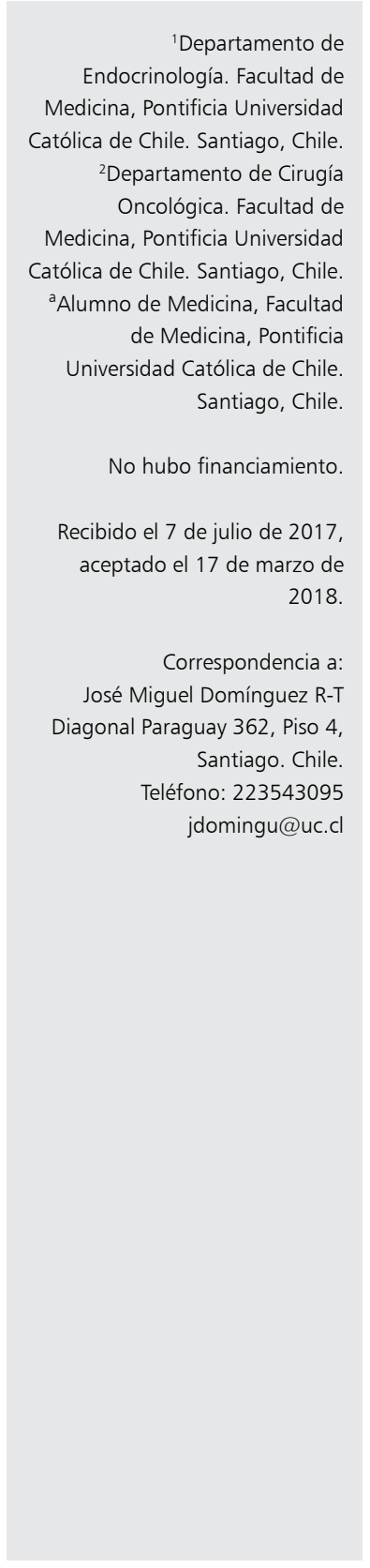

\section{Riesgo de recurrencia en cáncer diferenciado de tiroides: escala MINSAL}

\author{
JOSÉ MIGUEL DOMÍNGUEZ1', MARÍA TERESA MARTÍNEZa, \\ JOSÉ MIGUEL MASSARDO ${ }^{a}$, SUELII MUÑOZa, NICOLÁS DROPPELMANN ${ }^{2}$, \\ HERNÁN E. GONZÁLEZ², LORENA MOSSO ${ }^{1}$
}

\section{Risk of recurrence in differentiated thyroid cancer}

Background: Differentiated thyroid cancer (DTC) is generally associated with a favorable prognosis. Its treatment requires surgery, selective use of radioiodine and levothyroxine, and its intensity must be adjusted to the initial risks of mortality and recurrence. Aim: To validate the risk of recurrence classification developed by the Chilean Ministry of Health in 2013 (MINSAL 2013), and compare it with the American Thyroid Association (ATA) 2009 and 2015 classifications. Material and Methods: Retrospective study of 362 patients with DTC aged $44.3 \pm 13.4$ years ( $84 \%$ women), treated with total thyroidectomy, selective radioiodine ablation and levothyroxine and followed for a median of 4.2 years (range 2.0-7.8). Risk of recurrence was estimated with MINSAL 2013, ATA 2009 and ATA 2015 classifications, and risk of mortality with $7^{\text {th }}$ and $8^{\text {th }}$ American Joint Committee on Cancer (AJCC)/TNM systems. Clinical data obtained during follow-up were used to detect structural and biochemical persistence/recurrence. Results: A mean dose of $104 \pm 48 \mathrm{mCi}$ radioiodine was received by $91 \%$ of patients. MINSAL 2013 classified 148 (41\%), 144 (40\%), 67 (19\%) and $3(1 \%)$ patients as very low, low, intermediate and high risk of recurrence, respectively. Forty-five (12.4\%) patients had persistence or recurrence during follow-up: 33 structural and 12 biochemical. Rates of persistence/recurrence on each category of MINSAL 2013 were $4.1 \%, 7.6 \%, 37.3 \%$ and $100 \%$, respectively $(p<0.01)$. Areas under Receiver Operating Characteristic curves for persistence or recurrence of MINSAL 2013, ATA 2009 and ATA 2015 were 0.77 vs 0.73 vs 0.72, respectively. Conclusions: MINSAL 2013 classifies appropriately DTC patients and estimates correctly their risk of persistence or recurrence.

(Rev Med Chile 2018; 146: 282-289)

Key words: Thyroid Neoplasms; Neoplasm Recurrence, Local; Predictive Value of Tests; Treatment Outcome.
E 1 cáncer diferenciado de tiroides (CDT) es la neoplasia endocrinológica más frecuente $^{1,2}$. El tratamiento consiste en cirugía, uso selectivo de radioyodo (RAI) y suplementación con levotiroxina; el pronóstico, en general, es favorable, asociado a una baja mortalidad global2,3. La extensión de la cirugía, la necesidad de RAI y la dosis de levotiroxina requeridos en cada paciente dependen de su riesgo individual de mortalidad y recurrencia ${ }^{3}$.

Con el objetivo de estimar el riesgo de recurrencia, en 2009, la American Thyroid Association (ATA) propuso y validó la escala ATA 2009, que clasifica a los pacientes en 3 categorías de riesgo: 
alto, intermedio y bajo, según las características clínicas, histológicas y de laboratorio (Anexo 1$)^{4,5}$. Tras su publicación, la información utilizada para confeccionar la escala ATA 2009 se ha complementado con diversos elementos, que permitieron el desarrollo de las escalas MINSAL 2013 y ATA 2015 (Anexo 1) 3,6. Entre los elementos considerados, destacan que el impacto del compromiso ganglionar en la recurrencia depende de la masa ganglionar comprometida y el rol cada vez menor de la extensión extratiroidea mínima (EETM) sobre el riesgo de recurrencia ${ }^{3,6,7}$.

A diferencia de ATA 2009, que considera la presencia de cualquier metástasis ganglionar como riesgo de recurrencia intermedio, MINSAL 2013 clasifica estos pacientes como riesgo bajo o intermedio, dependiendo de la masa ganglionar

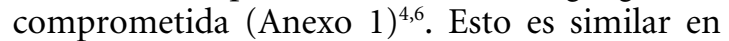
el caso de ATA 2015, que, incluso, considera la presencia de metástasis ganglionares cervicales mayores a $3 \mathrm{~cm}$ de diámetro como riesgo de recurrencia alto $^{3}$. En relación a la EETM, MINSAL 2013 es consistente con las últimas series publicadas, y no le confiere un aumento en el riesgo de recurrencia, mientras que tanto ATA 2009 como ATA 2015 clasifican su presencia como riesgo de recurrencia intermedio ${ }^{8,9}$.

El menor impacto de la EETM y el efecto variable del compromiso ganglionar de acuerdo a la masa tumoral involucrada motivaron, también, la actualización del sistema de clasificación de la American Joint Committe on Cancer (TNM/AJCC), que estima mortalidad atribuida al CDT. La octava versión del AJCC/TNM redujo el impacto de la EETM y del compromiso ganglionar sobre la mortalidad y aumentó la edad que determina peor pronóstico de 45 a 55 años ${ }^{10,11}$. De esta forma, se evita la "sobreestadificación” de la séptima versión de AJCC/TNM, que clasificaba, por ejemplo, a todos los pacientes mayores de 45 años con microcarcinoma papilar con EETM como etapa III, en circunstancias que la mortalidad asociada a esa enfermedad es menor a $2 \%$, compatible con etapa I, como lo clasifica la octava versión de AJCC/TNM ${ }^{11}$.

Nuestra hipótesis es que MINSAL 2013 predice correctamente el riesgo de recurrencia del CDT. El objetivo general de este trabajo es describir la forma de presentación y la evolución de los pacientes con CDT tratados en nuestra institución, determinar las tasas de persistencia/recurrencia y clasificar los pacientes según ATA 2009, MINSAL
2013 y ATA 2015. El objetivo específico de este estudio es validar MINSAL 2013 y comparar su rendimiento con ATA 2009 y ATA 2015.

\section{Métodos}

\section{Sujetos}

Tras la aprobación del Comité de Ética de Investigación de nuestra institución, se realizó un estudio de registros retrospectivo, que incluyó a 362 pacientes de $\geq 18$ años de edad con diagnóstico de CDT, sometidos a tiroidectomía total, entre 2009 y 2013, que recibieron o no RAI, seguidos por al menos dos años.

Se realizó disección linfática en los pacientes en que se certificó compromiso linfonodal clínico, definido como presencia de metástasis linfáticas al examen físico, en la ecografía cervical preoperatoria, en el estudio citohistológico preoperatorio o durante la cirugía. No se realizó disección linfática profiláctica. Todos los pacientes que recibieron RAI siguieron dieta restringida en yodo por 2 semanas previo a su administración y se certificó la presencia de hormona tiroestimulante $(\mathrm{TSH}) \geq 30$ $\mathrm{uUI} / \mathrm{mL}$, sí fueron preparados con suspensión de levotiroxina, o se administró TSH recombinante humana de acuerdo al protocolo sugerido en guías clínicas $^{3,4,6}$. La indicación de RAI fue a discreción del médico tratante, siguiendo las guías de la ATA 2009 y las guías locales de nuestra institución ${ }^{4}$. Se realizó exploración sistémica entre 5 y 7 días después de la administración de RAI en todos los pacientes que lo recibieron. Considerando los hallazgos de la ecografía preoperatoria e intraoperatorios, el estudio histológico y el resultado de la exploración sistémica en los pacientes que recibieron RAI, se clasificó a los pacientes con las escalas ATA 2009, ATA 2015 y MINSAL 2013 (Anexo 1), y se estadificaron de acuerdo a las la $7^{\circ}$ y $8^{\circ}$ ediciones del sistema de estadificación de la AJCC/UICC (etapas I, II, III y IV) ${ }^{3,11}$.

\section{Seguimiento}

Tras el tratamiento inicial, todos los pacientes recibieron terapia con levotiroxina y mantuvieron $\mathrm{TSH}<1,0 \mathrm{uUI} / \mathrm{mL}$, se realizaron al menos 2 ecografías cervicales y 2 mediciones de tiroglobulina $(\mathrm{Tg})$ y anticuerpos antitiroglobulina (AcTg) séricos, suprimidos o estimulados por suspensión de levotiroxina o TSH recombinante. 
Los pacientes fueron controlados con $\mathrm{Tg}, \mathrm{AcTg}$ y ecografía cervical cada 6 meses durante el primer año y, luego, cada 6-12 meses a discreción del médico tratante.

\section{Eventos clínicos registrados}

Se consideró recurrencia o persistencia de enfermedad estructural la presencia de imágenes sospechosas en la ecografía cervical con estudio citológico compatible con CDT, o de imágenes extracervicales compatibles en otros estudios (tomografía axial computada o resonancia magnética), o imágenes funcionales (exploración sistémica diagnóstica con ${ }^{131} \mathrm{I}$ o ${ }^{18} \mathrm{FDG}$ PET-CT), realizados a solicitud del médico tratante.

Se consideró recurrencia o persistencia de enfermedad bioquímica la presencia de $\mathrm{Tg}$ basal $\geq 1 \mathrm{ng} / \mathrm{ml}$ o Tg estimulada por $\mathrm{TSH} \geq 2 \mathrm{ng} / \mathrm{ml}$, en ausencia de enfermedad estructural.

Al final del seguimiento, la ausencia de imágenes anormales en estudios radiológicos, la presencia de Tg basal $<1,0 \mathrm{ng} / \mathrm{dL}$ o Tg estimulada $<2,0 \mathrm{ng} / \mathrm{dL}$, se consideró ausencia de enfermedad.

\section{Laboratorio}

La Tg se midió por inmunoensayo (Immulite 2000, Siemens, Los Angeles, CA, USA) con sensibilidad funcional de $0,9 \mathrm{ng} / \mathrm{mL}$. Los AcTg se midieron por inmunoensayo (Architect i1000, Laboratories, Abbott Park, IL, USA) (valor de referencia del fabricante hasta 4,11 IU/mL). A partir del 12 de mayo de 2014, se midió Tg de segunda generación (Tg-2G) mediante otro inmunoensayo (Elecsys II, Roche Diagnostics, Rotkreuz, Suiza) con sensibilidad funcional de $0,1 \mathrm{ng} / \mathrm{mL}$. Tanto la Tg como la $\mathrm{Tg}-2 \mathrm{G}$ fueron normalizadas con CRM 457.

\section{Análisis estadístico}

Para el análisis de datos las variables continuas se presentan como media \pm desviación estándar, o mediana y rango, según corresponda; y las variables nominales como frecuencias y porcentajes. La capacidad individual de las variables para predecir recurrencia se estudió por análisis univariado y la capacidad grupal de la combinación de ellas con regresión logística. Se comparó el área bajo la curva ROC de las escalas ATA 2009, ATA 2015 y MINSAL 2013 con la prueba de De Long. Se consideró significancia estadística con un valor $\mathrm{p}<0,05$. El análisis estadístico se realizó con los programa SPSS (versión 15.0.0: SPSS, Inc., Chi- cago, IL, USA) y MedCalc para Windows (versión 17.9.5, MedCalc Software, Ostende, Bélgica).

\section{Resultados}

Se incluyeron 362 pacientes, $303(83,7 \%)$ mujeres, de 44,3 $\pm 13,4$ años, seguidos por una mediana de 4,2 años (rango 2,0-7,8); 91\% de los pacientes recibió RAI (dosis $104 \pm 48 \mathrm{mCi}$ ). Como se detalla en la Tabla 1, la mayoría de los pacientes fue clasificada como riesgo de recurrencia bajo o muy bajo: las escalas ATA 2009, ATA 2015 y MINSAL 2013 clasificaron como riesgo bajo o muy bajo a $56,9 \%, 64,6 \%$ y $80,7 \%$, respectivamente. (Tabla 1).

Tabla 1. Características de los pacientes

\begin{tabular}{|c|c|}
\hline & $(n=362)$ \\
\hline $\begin{array}{l}\text { Histología } \\
\text { Cáncer papilar (CPT) } \\
\text { Cáncer folicular (CFT) } \\
\text { CPT + CFT }\end{array}$ & $\begin{array}{r}345(95,3 \%) \\
7(1,9 \%) \\
10(2,8 \%)\end{array}$ \\
\hline $\begin{array}{l}\text { AJCC 7a Edición } \\
\text { I } \\
\text { II } \\
\text { III } \\
\text { IVA } \\
\text { IVB } \\
\text { IVC }\end{array}$ & $\begin{array}{rr}284 & (78,5 \%) \\
13 & (3,6 \%) \\
48 & (13,3 \%) \\
15 & (4,1 \%) \\
0 & \\
2 & (0,6 \%)\end{array}$ \\
\hline $\begin{array}{l}\text { AJCC 8a Edición } \\
\text { I } \\
\text { II } \\
\text { III } \\
\text { IVA } \\
\text { IVB } \\
\text { IVC }\end{array}$ & $\begin{array}{rr}334 & (92,3 \%) \\
25 & (6,9 \%) \\
1 & (0,3 \%) \\
0 & \\
2 & (0,6 \%) \\
0 & \end{array}$ \\
\hline $\begin{array}{l}\text { ATA } 2009 \\
\text { Bajo } \\
\text { Intermedio } \\
\text { Alto }\end{array}$ & $\begin{array}{r}206(56,9 \%) \\
153(42,3 \%) \\
3(0,8 \%)\end{array}$ \\
\hline $\begin{array}{l}\text { ATA } 2015 \\
\text { Bajo } \\
\text { Intermedio } \\
\text { Alto }\end{array}$ & $\begin{array}{r}234(64,6 \%) \\
125(24,5 \%) \\
3(0,8 \%)\end{array}$ \\
\hline $\begin{array}{l}\text { MINSAL } 2013 \\
\text { Muy bajo } \\
\text { Bajo } \\
\text { Intermedio } \\
\text { Alto }\end{array}$ & $\begin{array}{c}148(40,9 \%) \\
144(39,8 \%) \\
67(18,5 \%) \\
3 \quad(0,8 \% \%)\end{array}$ \\
\hline $\begin{array}{l}\text { Persistencia/Recurrencia } \\
\text { No } \\
\text { Bioquímica } \\
\text { Estructural }\end{array}$ & $\begin{array}{rr}317 & (87,6 \%) \\
12 & (3,3 \%) \\
33 & (9,1 \%)\end{array}$ \\
\hline
\end{tabular}


Cuando se compararon los sistemas de clasificación TNM/AJCC destacó un aumento de la proporción de pacientes clasificados como etapa I: de $78 \%$ en la $7^{\circ}$ versión a $92,3 \%$ en la $8^{\circ}$ versión. Asimismo, en la $8^{\circ}$ versión disminuyó el porcentaje de pacientes clasificados como etapa III (13,3\% vs $0,3 \%$ ) (Tabla 1 ).

Se encontró recurrencia/persistencia en 45 pacientes (12,4\%): 33 estructural y bioquímica en 12 pacientes (Tabla 1 ). De los 317 pacientes que no tuvieron persistencia/recurrencia, se midió Tg-2G en 241: 233 (97\%) tenían Tg $\leq 0,2$ ng/dL. De los 33 pacientes con enfermedad estructural, 28 (85\%) tuvieron adenopatías cervicales, 3 (9\%) metástasis pulmonares y $2(6 \%)$ metástasis pulmonares y óseas. Dos pacientes del total de la serie fallecieron específicamente por CDT. La Tabla 2 muestra que las tasas de persistencia/recurrencia fueron significativamente distintas en las diferentes categorías establecidas por las 3 escalas ( $\mathrm{p}<0,01)$. La escala MINSAL 2013 tuvo una mayor área bajo la curva ROC que ATA $2009(0,77$ vs 0,$72 ; p=0,02)$ y similar a ATA $2015(0,77$ vs 0,$73 ; \mathrm{p}=0,06)$. El área bajo las curvas ROC de ATA 2009 y ATA 2015 fue similar $(\mathrm{p}=0,62)$.

$\mathrm{Al}$ analizar las tasas de persistencia/recurrencia estructural, que es el evento clínico más importante en pacientes con CDT, se mantuvo la diferencia de áreas bajo la curva ROC entre MINSAL 2013 y ATA $2009(0,74$ vs 0,$69 ; \mathrm{p}=0,04)$, pero no hubo diferencias entre MINSAL 2013 y ATA 2015 (0,74 vs 0,$71 ; \mathrm{p}=0,3)$ ni entre ATA 2009 y ATA 2015 $(0,69$ vs 0,$71 ; \mathrm{p}=0,12)$ (Tabla 3$)$.

\section{Discusión}

Este estudio evalúa la capacidad de la escala MINSAL 2013 para clasificar la recurrencia en pacientes con CDT y permite concluir que esta escala clasifica correctamente los pacientes con CDT de acuerdo a su riesgo de recurrencia. Nuestra serie incluye pacientes con CDT con un perfil similar a la mayoría de las series nacionales e internacionales: amplio predominio de mujeres y la mayoría de los casos tiene CDT de riesgo bajo o muy bajo de recurrencia ${ }^{12}$. Aunque todas las escalas lograron recurrencias distintas entre las categorías en que clasificaron a los pacientes con CDT, la discreta mayor área bajo la curva ROC de MINSAL 2013, respecto a ATA 2009 y ATA 2015,
Tabla 2. Clasificación de los pacientes según riesgo de recurrencia de acuerdo a las escalas ATA 2009, ATA 2015 y MINSAL 2013

\begin{tabular}{|lrcr|}
\hline Riesgo & \multicolumn{1}{c|}{$\begin{array}{c}\text { Total } \\
\mathbf{n}(\%)\end{array}$} & $\begin{array}{c}\text { Recurrencia } \\
\mathbf{n}(\%)\end{array}$ & \\
ATA 2009 & & & \\
Bajo & $206(56,9 \%)$ & $9(4,4 \%)$ & $\mathrm{p}<0,001$ \\
Intermedio & $153(42,3 \%)$ & $33(21,6 \%)$ & \\
Alto & $3(0,8 \%)$ & $3(100 \%)$ & \\
ATA 2015 & & & \\
Bajo & $234(64,6 \%)$ & $12(5,1 \%)$ & $\mathrm{p}<0,001$ \\
Intermedio & $125(34,5 \%)$ & $30(24,0 \%)$ & \\
Alto & $3(0,8 \%)$ & $3(100 \%)$ & \\
MINSAL 2013 & & & \\
Muy Bajo & $148(40,9 \%)$ & $6(4,1 \%)$ & $\mathrm{p}<0,001$ \\
Bajo & $144(39,8 \%)$ & $11(7,6 \%)$ & \\
Intermedio & $67(18,5 \%)$ & $25(37,3 \%)$ & \\
Alto & $3(0,8 \%)$ & $3(100 \%)$ & \\
\hline
\end{tabular}

$p<0,01$ para tendencia en la comparación de frecuencias de recurrencia entre las categorías de las tres escalas estudiadas.

\section{Tabla 3. Tasas de persistencia/recurrencia estructural según riesgo de recurrencia de acuerdo a las escalas ATA 2009, ATA 2015 y MINSAL 2013}

\begin{tabular}{|lccc|}
\hline & ATA 2009 & ATA 2015 & MINSAL 2013 \\
\hline Muy Bajo & NA & NA & $3,5 \%$ \\
Bajo & $3,3 \%$ & $3,2 \%$ & $3,4 \%$ \\
\hline Intermedio & $11,8 \%$ & $14,1 \%$ & $21,2 \%$ \\
\hline Alto & $100 \%$ & $100 \%$ & $100 \%$ \\
\hline
\end{tabular}

$p<0,01$ para tendencia en la comparación de frecuencias de recurrencia entre las categorías de las tres escalas estudiadas. NA = no aplica.

sugiere un mejor rendimiento diagnóstico para predecir persistencia/recurrencia de CDT.

A diferencia de las escalas desarrolladas por la ATA, que se componen de 3 categorías de riesgo: bajo, intermedio y alto, MINSAL 2013 consta de 4 categorías de recurrencia: muy bajo, bajo, intermedio y alto $^{3,4,6}$. La categoría de riesgo 
muy bajo de recurrencia incluye pacientes con microcarcinomas diferenciados intratiroideos, cuyo diámetro no supera $1 \mathrm{~cm}$, y los carcinomas foliculares mínimamente invasores de hasta $2 \mathrm{~cm}$, que no presentan invasión vascular venosa, que en nuestra serie y en otras previamente publicadas se asocian a tasas de recurrencia menores a $5 \%{ }^{3}$. La ventaja de incluir esta categoría es la identificación de los pacientes de mejor pronóstico, que no requieren intervenciones adicionales como ablación con RAI ni supresión de $\mathrm{TSH}^{13,14}$. La importancia de este punto radica en que, además de no mejorar el pronóstico de estos pacientes, estas terapias tienen efectos adversos. En el caso del RAI, incluso, la administración de dosis bajas se ha asociado a sialoadenitis, obstrucción del conducto lagrimal, alteraciones del sentido del gusto y manifestaciones clínicas del hipotiroidismo o costo económico del rhTSH requerido para su administración, entre otros ${ }^{15}$. En relación a la supresión de TSH, el hipertiroidismo subclínico es un reconocido factor de riesgo para arritmias, osteoporosis y fracturas, particularmente en mujeres postmenopáusicas ${ }^{16}$.

Al comparar la clasificación de los pacientes por las distintas escalas de riesgo de recurrencia, se evidencia que las modificaciones realizadas a ATA 2009 aumentaron la proporción de pacientes clasificados como riesgo de recurrencia bajo o muy bajo: subió de 56,9\% en ATA 2009 a $64,6 \%$ y $80,7 \%$ en ATA 2015 y MINSAL 2013, respectivamente. Este cambio en la clasificación no subestimó el riesgo de recurrencia de los pacientes incluidos en las categorías de riesgo bajo o muy bajo, porque se mantuvo cercano $5 \%$, similar a lo encontrado en esta serie y en otras publicadas para ATA 2009,5,17. Sin embargo, el cambio en la clasificación de los pacientes sí implicó una mejor selección de los pacientes de riesgo intermedio, especialmente por MINSAL 2013, que clasificó a 18,5\% de los pacientes en esta categoría, de los cuales $37,3 \%$ tuvo persistencia/recurrencia.

En relación a la clasificación de los pacientes por los sistemas AJCC/TNM, destaca un au- mento de los pacientes clasificados como etapa I: de $78,5 \%$ a $92,3 \%$ en las versiones $7^{\circ}$ y $8^{\circ}$, respectivamente. La baja mortalidad de nuestra serie impide comparar las tasas de mortalidad entre las distintas etapas AJCC/TNM, pero series previas validan los cambios realizados en la última versión de este sistema, pues se reportan sobrevidas a 10 años de $99 \%$ y sobre $95 \%$ en las etapas I y II de $\mathrm{CDT}^{11}$. Tal como ocurre con las modificaciones de las escalas de recurrencia, el objetivo de estos cambios es reducir la "sobreestadificación" y el sobretratamiento de los pacientes con CDT.

Nuestro estudio tiene algunas limitaciones. Una de ellas es el tiempo de seguimiento. Sin embargo, aunque el CDT puede recurrir tardíamente, se reconoce que cerca de $50 \%$ y hasta $80 \%$ de las recurrencias ocurren los primeros 3 y 5 años luego del manejo inicial, respectivamente ${ }^{18}$. Adicionalmente, destaca que de los 317 pacientes que no tuvieron persistencia/recurrencia según los criterios establecidos, se midió Tg-2G en 241 de ellos, y $97 \%$ tenían $\mathrm{Tg} \leq 0,2 \mathrm{ng} / \mathrm{dL}$, lo que es consistente con respuesta excelente a tratamiento y se asocia a riesgo de recurrencia estructural menor a $2 \%{ }^{3}$.

Otra limitación de este estudio son los cambios en las indicaciones de ablación con RAI. Estudios publicados en la última década recomiendan un uso más selectivo y dosis menores de RAI, determinado por su escaso o nulo efecto sobre la mortalidad y recurrencia, especialmente en los pacientes de riesgo bajo o muy bajo, que constituyen $80 \%$ de nuestra serie $e^{14,19,20}$. De esta forma, en la actualidad un porcentaje mayor de los pacientes incluidos en este estudio no recibiría RAI y, en caso de indicarlo, serían dosis menores.

En conclusión, nuestra serie demuestra que la escala MINSAL 2013 clasifica adecuadamente los pacientes con CDT, estima de manera correcta el riesgo de recurrencia en pacientes con CDT tratados con tiroidectomía total y sugiere que MINSAL 2013 podría tener mejor rendimiento que ATA 2009 y ATA 2015. 
Anexo 1. Detalle de las escalas para riesgo de recurrencia American Thyroid Association (ATA) 2009, MINSAL 2013 y ATA 2015

\section{Escala ATA 2009}

\begin{tabular}{|c|c|c|}
\hline Riesgo bajo & Riesgo intermedio & Riesgo alto \\
\hline $\begin{array}{l}\text { Presencia de todas estas condiciones: } \\
\text { - Ausencia de metástasis locales o a } \\
\text { distancia } \\
\text { - Resección tumoral completa } \\
\text { - Ausencia de invasión loco-regional } \\
\text { - Ausencia de histología agresiva o } \\
\text { invasión vascular } \\
\text { - Si recibe I }{ }^{131} \text {, ausencia de captación } \\
\text { fuera del lecho tiroideo en la explo- } \\
\text { ración sistémica }\end{array}$ & $\begin{array}{l}\text { Presencia de cualquiera de las siguien- } \\
\text { tes: } \\
\text { - Extensión extra-tiroidea microscópica } \\
\text { - Captación de } 1131 \text { en ganglios } \\
\text { cervicales o cervical fuera del lecho } \\
\text { tiroideo en la exploración sistémica } \\
\text { - Histología agresiva }\end{array}$ & $\begin{array}{l}\text { Presencia de cualquiera de las siguien- } \\
\text { tes: } \\
\text { - Extensión extratiroidea macroscópica } \\
\text { - Resección tumoral incompleta } \\
\text { - Metástasis a distancia } \\
\text { - Tiroglobulina pre ablativa inapropia- } \\
\text { damente alta }\end{array}$ \\
\hline
\end{tabular}

\section{Escala MINSAL 2013}

\begin{tabular}{|c|c|c|c|}
\hline Riesgo muy bajo & Riesgo bajo & Riesgo intermedio & Riesgo alto \\
\hline $\begin{array}{l}\text { - Microcarcinoma papilar } \\
\text { de tiroides unifocal } \\
\text { - Microcarcinoma papilar } \\
\text { de tiroides multifocal, } \\
\text { cuya sumatoria de focos } \\
\text { es } \leq 2 \mathrm{~cm} \\
\text { - Carcinoma folicular de } \\
\text { tiroides mínimamente } \\
\text { invasor, } \leq 2 \mathrm{~cm} \text {, sólo } \\
\text { invasión microscópica } \\
\text { capsular } \\
\text { Deben cumplirse además to- } \\
\text { dos los requisitos siguientes: } \\
\text { - Ausencia de extensión } \\
\text { extratiroidea, varieda- } \\
\text { des histológicas de alto } \\
\text { riesgo, invasión vascular, } \\
\text { compromiso ganglionar y } \\
\text { metástasis a distancia } \\
\text { - Resección completa del } \\
\text { tumor }\end{array}$ & $\begin{array}{l}\text { - Microcarcinoma papilar } \\
\text { de tiroides multifocal (si } \\
\text { la sumatoria de focos es } \\
>2 \mathrm{~cm} \text { ) } \\
\text { - Cáncer papilar de tiroides, } \\
\text { diámetro >1-4 cm } \\
\text { (T1b-T2) } \\
\text { - Ausencia de variedades } \\
\text { histológicas asociadas a } \\
\text { mayor agresividad (células } \\
\text { altas, insular, columnar, } \\
\text { esclerosante difuso, etc.) } \\
\text { - Ausencia de extensión } \\
\text { extratiroidea, o presencia } \\
\text { de extensión extratiroidea } \\
\text { mínima, reportado sólo } \\
\text { en el informe histológico } \\
\text { que no invade tráquea, } \\
\text { nervio laríngeo recurrente } \\
\text { ni esófago } \\
\text { - Ausencia de invasión } \\
\text { vascular venosa } \\
\text { - Compromiso de hasta } \\
5 \text { ganglios, } 5 \text { mm } \\
\text { de diámetro mayor, sin } \\
\text { extensión extranodal } \\
\text { - Ausencia de metástasis a } \\
\text { distancia } \\
\text { - Resección completa del } \\
\text { tumor } \\
\text { - Cáncer folicular de tiroi- } \\
\text { des mínimamente invasor } \\
\text { (solo cápsula) } 2 \text {-4 cm }\end{array}$ & $\begin{array}{l}\text { - Cáncer papilar de tiroides } \\
\text { con diámetro mayor } \\
>4 \text { cm } \\
\text { - Compromiso ganglionar } \\
\text { clínico (evidenciado al } \\
\text { examen físico preope- } \\
\text { ratorio, en estudio con } \\
\text { imágenes preoperatorias } \\
\text { o durante la cirugía) o, } \\
\text { - Compromiso ganglionar } \\
\text { patológico, si la metásta- } \\
\text { sis más grande es mayor } \\
\text { a } 5 \text { mm, si hay más de } 5 \\
\text { ganglios o si existe exten- } \\
\text { sión extranodal } \\
\text { - Presencia de variedades } \\
\text { histológicas asociadas } \\
\text { a mayor agresividad } \\
\text { (variedad de células altas, } \\
\text { columnar, esclerosante, } \\
\text { etc.) } \\
\text { - Presencia de extensión ex- } \\
\text { tratiroidea macroscópica } \\
\text { (evidenciada en cirugía), } \\
\text { sin invasión de esófago, } \\
\text { tráquea o nervio laríngeo } \\
\text { recurrente } \\
\text { - Presencia de invasión } \\
\text { vascular venosa } \\
\text { - Cáncer folicular de } \\
\text { tiroides no mínimamente } \\
\text { invasor }\end{array}$ & $\begin{array}{l}\text { Puede cumplir cualquiera de } \\
\text { estas características: } \\
\text { - Presencia de extensión } \\
\text { extratiroidea macroscópi- } \\
\text { ca, evidenciada durante } \\
\text { la cirugía invadiendo } \\
\text { esófago, tráquea o nervio } \\
\text { laríngeo recurrente (T4a) } \\
\text { y enfermedad muy avan- } \\
\text { zada que invade fascia } \\
\text { prevertebral y compro- } \\
\text { mete la arteria carótida o } \\
\text { vasos mediastínicos (T4b) } \\
\text { - Cáncer pobremente dife- } \\
\text { renciado } \\
\text { - Presencia de metástasis a } \\
\text { distancia } \\
\text { - Resección Tumoral incom- } \\
\text { pleta (R1 o R2) } \\
\text { - Tiroglobulina pre ablativa } \\
\text { inapropiadamente alta } \\
>50 \text { ng/mL }\end{array}$ \\
\hline
\end{tabular}




\section{Escala ATA 2015}

\begin{tabular}{|c|c|c|}
\hline Riesgo bajo & Riesgo intermedio & Riesgo alto \\
\hline - Cáncer papilar con todas estas & \multirow{12}{*}{$\begin{array}{l}\text { Presencia de cualquiera de las siguien- } \\
\text { tes: } \\
\text { - Extensión extra-tiroidea microscópica } \\
\text { - Captación de } 1131 \text { cervical en la } \\
\text { exploración sistémica } \\
\text { - Histología agresiva (células altas, } \\
\text { variante hobnail, células columna- } \\
\text { res, etc.) } \\
\text { - Cáncer papilar con invasión vascular } \\
\text { - Compromiso ganglionar clínico o } \\
\text { más de } 5 \text { ganglios de hasta } 3 \text { cm } \\
\text { - Microcarcinoma papilar con ex- } \\
\text { tensión extratiroidea y BRAFV600E } \\
\text { mutado (si se sabe) }\end{array}$} & Presencia de cualquiera de las siguien- \\
\hline Resección tumoral completa & & - Invasión extratiroidea macroscópica \\
\hline & & - Resección tumoral incompleta \\
\hline distancia, invasión loco-regional, his- & & - Metástasis a distancia \\
\hline tología agresiva o invasión vascular & & Tirgolabulinz córica nect onometori \\
\hline Si recibe $I^{131}$, ausencia de captación & & sugerente de metástasis a distancia \\
\hline ración sistémica & & - Compromiso ganglionar con nódulos \\
\hline Compromiso ganglionar ausente o & & 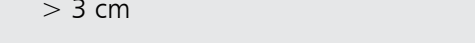 \\
\hline hasta 5 ganglios con micrometástasis & & - Cáncer folicular con más de 4 focos \\
\hline $\begin{array}{l}\text { - Cáncer papilar variedad folicular en- } \\
\text { capsulado, intratiroideo }\end{array}$ & & \\
\hline $\begin{array}{l}\text { - Cáncer folicular intratiroideo, con } \\
\text { hasta } 4 \text { focos de invasión vascular }\end{array}$ & & \\
\hline $\begin{array}{l}\text { - Microcarcinoma papilar intratiroi- } \\
\text { deo, uni o multifocal }\end{array}$ & & \\
\hline
\end{tabular}

\section{Referencias}

1. Davies L, Welch HG. Current thyroid cancer trends in the United States. JAMA Otolaryngol Head Neck Surg 2014; 140 (4): 317-22.

2. Carling T, Udelsman R. Thyroid cancer. Annu Rev Med 2014; 65: 125-37.

3. Haugen B, Alexander E, Bible K, Doherty G, Mandel S, Nikiforov Y, et al. 2015 American Thyroid Association Management Guidelines for Adult Patients with Thyroid Nodules and Differentiated Thyroid Cancer. Thyroid 2016; 26: 1-33.

4. American Thyroid Association Guidelines Taskforce on Thyroid N, Differentiated Thyroid C, Cooper DS, Doherty GM, Haugen BR, Kloos RT, et al. Revised American Thyroid Association management guidelines for patients with thyroid nodules and differentiated thyroid cancer. Thyroid 2009; 19 (11): 1167-214.

5. Tuttle RM, Tala H, Shah J, Leboeuf R, Ghossein R, Gonen $\mathrm{M}$, et al. Estimating risk of recurrence in differentiated thyroid cancer after total thyroidectomy and radioactive iodine remnant ablation: using response to therapy variables to modify the initial risk estimates predicted by the new American Thyroid Association staging system. Thyroid 2010; 20 (12): 1341-9.

6. Wohllk N, Pineda P, Tala H, Domínguez JM, González H, Amaral H, et al. Guía Clínica Nódulo Tiroideo y
Cáncer Diferenciado de Tiroides. Ministerio de Salud. Chile 2013; 1-110.

7. Randolph GW, Duh QY, Heller KS, LiVolsi VA, Mandel SJ, Steward DL, et al. The prognostic significance of nodal metastases from papillary thyroid carcinoma can be stratified based on the size and number of metastatic lymph nodes, as well as the presence of extranodal extension. Thyroid 2012; 22 (11): 1144-52.

8. Hay ID, Johnson TR, Thompson GB, Sebo TJ, Reinalda MS. Minimal extrathyroid extension in papillary thyroid carcinoma does not result in increased rates of either cause-specific mortality or postoperative tumor recurrence. Surgery 2016; 159 (1): 11-9.

9. Shin JH, Ha TK, Park HK, Ahn MS, Kim KH, Bae KB, et al. Implication of minimal extrathyroidal extension as a prognostic factor in papillary thyroid carcinoma. Int J Surg 2013; 11 (9): 944-7.

10. Nixon IJ, Wang LY, Migliacci JC, Eskander A, Campbell MJ, Aniss A, et al. An International Multi-Institutional Validation of Age 55 Years as a Cutoff for Risk Stratification in the AJCC/UICC Staging System for Well-Differentiated Thyroid Cancer. Thyroid 2016; 26 (3): 373-80.

11. Tarasova VD, Tuttle RM. Current Management of Low Risk Differentiated Thyroid Cancer and Papillary Microcarcinoma. Clin Oncol (R Coll Radiol) 2017.

12. Sapunar J, Muñoz S, Roa JC. Epidemiología del cáncer 
de tiroides en Chile. Resultados del estudio INCATIR. Rev Med Chile 2014; 142 (9): 1099-105.

13. Hay ID, Hutchinson ME, González-Losada T, McIver B, Reinalda ME, Grant CS, et al. Papillary thyroid microcarcinoma: a study of 900 cases observed in a 60-year period. Surgery 2008; 144 (6): 980-7; discussion 7-8.

14. Lamartina L, Durante C, Filetti S, Cooper DS. Low-risk differentiated thyroid cancer and radioiodine remnant ablation: a systematic review of the literature. J Clin Endocrinol Metab 2015; 100 (5): 1748-61.

15. Florenzano P, Guarda FJ, Jaimovich R, Droppelmann N, González H, Domínguez JM. Radioactive Iodine Administration Is Associated with Persistent Related Symptoms in Patients with Differentiated Thyroid Cancer. Int J Endocrinol 2016; 2016: 2586512.

16. Ross DS, Burch HB, Cooper DS, Greenlee MC, Laurberg P, Maia AL, et al. American Thyroid Association Guidelines for Diagnosis and Management of Hyperthyroidism and Other Causes of Thyrotoxicosis. Thyroid.
2016; 26 (10): 1343-421.

17. Pitoia F, Bueno F, Urciuoli C, Abelleira E, Cross G, Tuttle RM. Outcomes of patients with differentiated thyroid cancer risk-stratified according to the American thyroid association and Latin American thyroid society risk of recurrence classification systems. Thyroid 2013; 23 (11): 1401-7.

18. Durante C, Montesano T, Torlontano M, Attard M, Monzani F, Tumino S, et al. Papillary thyroid cancer: time course of recurrences during postsurgery surveillance. J Clin Endocrinol Metab 2013; 98 (2): 636-42.

19. Schlumberger M, Catargi B, Borget I, Deandreis D, Zerdoud S, Bridji B, et al. Strategies of radioiodine ablation in patients with low-risk thyroid cancer. N Engl J Med 2012; 366 (18): 1663-73.

20. Mallick U, Harmer C, Yap B, Wadsley J, Clarke S, Moss $\mathrm{L}$, et al. Ablation with low-dose radioiodine and thyrotropin alfa in thyroid cancer. N Engl J Med 2012; 366 (18): 1674-85. 